\title{
Prognostic Value of Myocardial Perfusion Imaging with a Cadmium-Zinc-Telluride SPECT Camera in Patients Suspected of Having Coronary Artery Disease
}

\author{
Elsemiek M. Engbers ${ }^{1,2}$, Jorik R. Timmer ${ }^{1}$, Mohamed Mouden ${ }^{1,2}$, Siert Knollema², Pieter L. Jager ${ }^{2}$, and \\ Jan Paul Ottervanger ${ }^{1}$ \\ ${ }^{1}$ Department of Cardiology, Isala, Zwolle, The Netherlands; and ${ }^{2}$ Department of Nuclear Medicine, Isala, Zwolle, The Netherlands
}

\begin{abstract}
The prognostic value of myocardial perfusion imaging (MPI) with the cadmium-zinc-telluride (CZT) SPECT camera is not well established. Therefore, the aim of the current study was to evaluate the prognostic value of MPI performed with a CZT SPECT camera in a large cohort of patients suspected of having coronary artery disease. Methods: Consecutive symptomatic stable patients $(n=$ $4,057)$ without a history of coronary artery disease underwent CZT SPECT MPI. During a median follow-up of 2.4 y (25th-75th percentile, 1.7-3.4), patients were monitored for primary (nonfatal myocardial infarction and cardiac mortality) and secondary outcomes (late revascularization [ $>90 \mathrm{~d}$ after scanning] and primary outcome). Results: Patients with normal perfusion demonstrated low annual event rates (primary outcome, $0.2 \%$; secondary outcome, $0.6 \%$ ). Annual event rates increased with the extent of abnormality of myocardial perfusion. In patients with small ischemic perfusion defects, annual event rates were $0.7 \%$ and $2.8 \%$ for the primary and secondary outcome, respectively. In patients with moderate or large ischemic perfusion defects, these event rates were $1.2 \%$ and $4.3 \%$, respectively. After multivariate analysis, the risk for events was significantly associated with the extent of ischemia (hazard ratio for small ischemic defects: 2.2, 95\% confidence interval [Cl], 0.9-5.9 and 4.6, $95 \% \mathrm{Cl}, 2.8-7.6$, for primary and secondary outcomes, respectively; hazard ratio for moderate or large ischemic defects: $4.0,95 \% \mathrm{Cl}$, 1.5-10.5 and $12.1,95 \% \mathrm{Cl}, 7.2-20.2$, for primary and secondary outcomes, respectively). Conclusion: Our findings show that MPI acquired with a CZT SPECT camera provides excellent prognostic information, with low event rates in patients with normal myocardial perfusion. In patients with abnormal SPECT MPI, the extent of abnormality is independently associated with an increased risk of events.
\end{abstract}

Key Words: prognosis; suspected coronary artery disease; SPECT; cadmium zinc telluride

J Nucl Med 2017; 58:1459-1463

DOI: 10.2967/jnumed.116.188516

\section{$\mathbf{S}$}

PECT myocardial perfusion imaging (MPI) is an established and widely available technique for the diagnostic and prognostic evaluation of patients suspected of having coronary artery disease

Received Dec. 16, 2016; revision accepted Feb. 28, 2017.

For correspondence or reprints contact: Elsemiek M. Engbers, Isala, Department of Cardiology, Dokter van Heesweg 2, 8025 AB Zwolle, The Netherlands.

E-mail: e.engbers@isala.nl

Published online Apr. 27, 2017.

COPYRIGHT (C 2017 by the Society of Nuclear Medicine and Molecular Imaging.
(CAD) (1). Guidelines for appropriate use criteria recommend SPECT MPI in patients with an intermediate pretest likelihood of CAD (2-4). Normal SPECT MPI is associated with an excellent prognosis, with annual cardiac event rates of less than $1 \%(5,6)$. In recent years, concerns about radiation exposure and time efficacy have led to the development of a new generation of $\gamma$-camera systems based on a novel detector technology using semiconductor detectors of cadmium-zinc-telluride (CZT). This new technology, with solid-state CZT detectors and multipinhole collimators focused on the heart, allows reduced radiation exposure and acquisition time without loss of image quality $(7,8)$. Although research about the diagnostic accuracy of these dedicated CZT SPECT cameras has been performed $(9,10)$, data on the prognostic value of SPECT imaging acquired using CZT detectors are scarce $(11,12)$. Therefore, the aim of the current study was to evaluate the prognostic value of MPI performed with a CZT SPECT camera in a large cohort of patients suspected of having CAD.

\section{MATERIALS AND METHODS}

\section{Study Population}

Patients referred for clinically indicated SPECT MPI in our hospital were prospectively enrolled in our registry. The study was approved by our local Medical Ethics committee and included a waiver of consent. For the current analysis, consecutive patients referred between May 2010 until June 2013 were included. Patients with a known history of CAD were excluded; no other exclusion criteria were applied. Information regarding the presence of risk factors was prospectively collected by written questionnaires. These data were verified and complemented with demographic and clinical information collected from medical records. All patients underwent initial lowdose ${ }^{99 \mathrm{~m}}$ Tc-tetrofosmin stress-first SPECT imaging. In the case of an abnormal stress perfusion, additional rest SPECT was performed. The pretest likelihood of CAD was assigned according to the criteria of Diamond and Forrester (13), with a risk threshold of less than $13.4 \%$ for low risk, between $13.4 \%$ and $87.2 \%$ for intermediate risk, and greater than $87.2 \%$ for high risk. The presence of left bundle branch block (LBBB) was assessed on an electrocardiogram obtained in the nuclear medicine department before stress testing. LBBB was defined as a QRS duration $0.12 \mathrm{~s}$ or more, with a broad-notched or slurred $\mathrm{R}$ wave in leads I, aVL, V5, and V6; absent Q waves in leads I, V5, and V6; and a R peak time more than $60 \mathrm{~ms}$ in leads V5 and V6 but normal in leads V1-V3 (14).

\section{Stress Procedures and Tracer Injection}

Stress testing was routinely performed with pharmacologic stress using adenosine $(140 \mu \mathrm{g} / \mathrm{min} / \mathrm{kg}$ for $6 \mathrm{~min})$, unless there was a contraindication for pharmacologic stress. Because of logistical reasons, 
pharmacologic stress testing with adenosine is common practice in our high-volume center. Patients were instructed to refrain from caffeinecontaining beverages for at least $24 \mathrm{~h}$ before the test. In the case of a contraindication for adenosine, patients underwent dobutamine (starting dose of $10 \mu \mathrm{g} / \mathrm{kg}$ per min, increased at 3-min intervals to a maximum of $50 \mu \mathrm{g} / \mathrm{kg}$ per min), regadenoson (fixed-dose of $400 \mu \mathrm{g}$ bolus injection over $15 \mathrm{~s}$ ), or bicycle testing. A weight-adjusted dose of 99m Tc-tetrofosmin (standard, $370 \mathrm{MBq}$; $500 \mathrm{MBq}$ for patients > $100 \mathrm{~kg}$ ) was administered after $3 \mathrm{~min}$ (adenosine), after $35 \mathrm{~s}$ (regadenoson), or when the target heart rate of greater than $85 \%$ of predicted maximal was reached (dobutamine, bicycle test). Patients scheduled for rest imaging received a dose of ${ }^{99 \mathrm{~m}} \mathrm{Tc}$-tetrofosmin (standard, 740 $\mathrm{MBq}$, but $1,000 \mathrm{MBq}$ for patients $>100 \mathrm{~kg}$ ).

\section{Myocardial Perfusion Image Acquisition}

Both stress and rest SPECT images were acquired 45-60 min after tracer injection. Time delay between the stress and rest studies was more than $3 \mathrm{~h}$ (15). All patients were imaged supine, with arms placed above the head. All patients were scanned with a CZT-based SPECT/CT camera (Discovery NM/CT 570c; GE Healthcare) with 19 stationary CZT detectors simultaneously imaging 19 cardiac views. Each detector comprised $32 \times 32$ pixelated $(2.46 \times 2.46 \mathrm{~mm})$ CZT elements. Acquisition time was $5 \mathrm{~min}$ for the stress images and $4 \mathrm{~min}$ for the rest images. This acquisition time was derived from the recommendations of the manufacturer, published experience, and our own qualitative assessment in heart phantom studies and our initial experience in patients (16). All SPECT studies were followed by an unenhanced low-dose CT scan during a breath-hold to provide the attenuation map for attenuation correction as previously described (17).

\section{Myocardial Perfusion Image Interpretation}

SPECT MPI was performed in an unmasked manner and semiquantitatively interpreted using a 17-segment model (18). Segments were scored by consensus of 2 experienced nuclear cardiology observers using a 5-point scoring system ( 0 , normal; 1 , equivocal; 2 , moderate; 3 , severe reduction of radioisotope uptake; 4 , absence of detectable tracer uptake) (19). The combination of attenuationcorrected and non-attenuation-corrected images was reviewed. A stress study was interpreted as normal if the summed stress scores were 3 or less (19). Additional rest SPECT was acquired if the stress images did not fulfill this criterion. The perfusion images were reviewed again after both stress and rest SPECT. An ischemic defect was defined as a summed difference score of 2 or greater (19). Reversible defects not fulfilling this criterion were assessed as equivocal for ischemia. Perfusion defects that demonstrated no reversibility were defined as fixed defects. SPECT was considered normal in the absence of reversible or fixed defects. Reversible perfusion defects constituting less than $10 \%$ of the myocardium were defined as small ischemic perfusion defects. Moderate/large ischemic perfusion defects were defined as reversible perfusion defects constituting greater than $10 \%$ of the myocardium. Fixed or reversible perfusion defects constituting less than $10 \%$ of the myocardium were defined as small total perfusion defects. Moderate/large total perfusion defects were defined as fixed or reversible perfusion defects constituting greater than $10 \%$ of the myocardium. Summed stress (total perfusion defect), summed rest, and difference scores (ischemic perfusion defect) were converted to percentage total myocardium (\% myocardium: summed scores/68 [maximum potential score $=4 \times 17] \times 100)$.

\section{Effective Radiation Dose}

The radiation dose for SPECT was calculated by multiplying the dose of ${ }^{99 \mathrm{~m}} \mathrm{Tc}$-tetrofosmin by $7.9 \mathrm{mSv} / \mathrm{GBq}$ as suggested by the International Commission on Radiological Protection (20).

\section{Follow-up}

Follow-up data were based on clinical visits or standardized telephone interviews. Patients were monitored for primary (nonfatal myocardial infarction $[\mathrm{MI}]$ and cardiac mortality) and secondary outcomes (late revascularization [ $>90 \mathrm{~d}$ after scanning] and primary outcome). Early elective revascularizations within $90 \mathrm{~d}$ after imaging were excluded from the survival analysis to eliminate events driven by imaging findings. Patients undergoing early revascularization were censored. Cardiac death was defined as death due to MI, significant cardiac arrhythmia, refractory heart failure, or cardiogenic shock. Sudden death occurring without another explanation was included as cardiac death. For the analysis of death from cardiac causes, we regarded death from other or unknown causes as censored observations. Nonfatal MI was defined using the criteria of typical chest pain, elevated cardiac enzyme levels, and typical changes on the electrocardiogram as defined by Thygesen et al. (21).

\section{Statistical Analysis}

Continuous variables are expressed as mean \pm SD or median (25th-75th percentile), and categoric variables are expressed as frequency (percentage). Differences between groups were assessed by the unpaired Student $t$ test, Mann-Whitney $U$ test, and $\chi^{2}$ test, where appropriate. The patient's pretest likelihood for CAD was determined with the standard Diamond criteria, with the assumption that chest pain was atypical (13). We calculated annualized event rates on the basis of events per patients-year and compared differences in annualized events between patients with different SPECT outcomes by Poisson regression for rate data. We used Cox proportional hazards regression to analyze the association between imaging result, patient characteristics, and clinical outcomes. Continuous predictors with a linear relationship were included in the model as a continuous variable, otherwise they were included as a dichotomous variable ( $>$ upper quartile). A $P$ value of less than 0.05 in univariate analysis was required for entry into the multivariate analysis. The proportional hazard assumption was evaluated graphically using log-log plots. Variables that did not demonstrate an effect on the outcome measure were not included in the Cox model. The multivariate analysis for the primary outcome included age, LBBB, body mass index (BMI), diabetes mellitus, and SPECT results. For the secondary outcome, age, sex, LBBB, diabetes mellitus, and SPECT results were included. Two-sided $P$ values of less than 0.05 were considered statistically significant in all tests. All statistical analysis was performed with a commercially available software package (SPSS, version 20.0 for Windows; SPSS); the Poisson regression for rate data was performed with Medcalc (version 16.2 for Windows; Medcalc).

\section{RESULTS}

\section{Study Population}

During a period of $3 \mathrm{y}$ and $1 \mathrm{mo}$, a total of 4,057 patients were included. The main indications for referral were atypical chest pain and dyspnea. Pretest likelihood was low in $9 \%$ of the patients and intermediate in $91 \%$ of the patients. The baseline characteristics are shown in Table 1 . The mean age of patients was $61 \pm 11 \mathrm{y}, 42 \%$ were men, and $13 \%$ were diabetic. Stress testing was performed with adenosine in $3,864(95 \%)$, with dobutamine in $103(3 \%)$, with regadenoson in $37(1 \%)$, and with exercise testing in $53(1 \%)$ of the patients.

\section{SPECT Results}

Of the total patient population, 3,137 (77\%) demonstrated normal SPECT MPI findings. Differences in baseline characteristics of patients with normal and abnormal SPECT MPI are 
TABLE 1

Baseline Characteristics of Total Patient Population

\begin{tabular}{|c|c|c|c|c|c|c|c|c|c|c|}
\hline Characteristic & $\begin{array}{l}\text { All patients } \\
(n=4,057)\end{array}$ & $\begin{array}{c}\text { Normal } \\
\text { SPECT } \\
(n=3,137)\end{array}$ & $\begin{array}{c}\text { Abnormal } \\
\text { SPECT } \\
(n=920)\end{array}$ & $P$ & $\begin{array}{c}\text { Small } \\
\text { ischemic } \\
\text { defect } \\
(n=353)\end{array}$ & $\begin{array}{l}\text { Moderate/ } \\
\text { large } \\
\text { ischemic } \\
\text { defect } \\
(n=222)\end{array}$ & $P$ & $\begin{array}{c}\text { Small total } \\
\text { defect } \\
(n=558)\end{array}$ & $\begin{array}{l}\text { Moderate/ } \\
\text { large } \\
\text { total defect } \\
(n=362)\end{array}$ & $P$ \\
\hline Age (y) & $61 \pm 11$ & $60 \pm 11$ & $64 \pm 11$ & $<0.001$ & $63 \pm 10$ & $64 \pm 10$ & 0.48 & $63 \pm 11$ & $64 \pm 11$ & 0.14 \\
\hline Male sex & $1,717(42)$ & 1,193 (38) & $524(57)$ & $<0.001$ & 188 (53.3) & 122 (55.0) & 0.69 & $306(55)$ & $218(60)$ & 0.11 \\
\hline $\mathrm{BMI}$ & $27.6 \pm 4.8$ & $27.3 \pm 4.6$ & $28.7 \pm 5.4$ & $<0.001$ & $29.5 \pm 5.3$ & $29.1 \pm 5.3$ & 0.39 & $28.9 \pm 5.3$ & $28.3 \pm 5.5$ & 0.11 \\
\hline Diabetes & $543(13)$ & 362 (12) & $181(20)$ & $<0.001$ & $83(23.5)$ & $50(22.5)$ & 0.78 & 108 (19) & $73(20)$ & 0.76 \\
\hline Hypertension & $2,444(60)$ & $1,861(60)$ & $583(63)$ & 0.03 & $247(70.0)$ & $140(63.1)$ & 0.09 & $366(66)$ & $217(60)$ & 0.08 \\
\hline Hypercholesterolemia & $1,706(42)$ & $1,299(41)$ & $407(44)$ & 0.13 & $167(47.3)$ & 118 (53.2) & 0.17 & $240(43)$ & $167(46)$ & 0.35 \\
\hline Current smoking & $644(16)$ & $484(15)$ & $160(17)$ & 0.16 & $70(19.8)$ & $33(14.9)$ & 0.13 & $96(17)$ & $64(18)$ & 0.86 \\
\hline Family history of CAD & $2,249(56)$ & $1,752(56)$ & $497(54)$ & 0.33 & $194(55.0)$ & $115(51.8)$ & 0.43 & $318(57)$ & $179(50)$ & 0.02 \\
\hline LBBB & $189(4.7)$ & $75(2.4)$ & $114(12.4)$ & $<0.001$ & $25(7.1)$ & $6(2.7)$ & 0.03 & $77(13.8)$ & $37(10.2)$ & 0.11 \\
\hline
\end{tabular}

Values are shown as number, with percentages in parentheses, or mean \pm SD. Data are a comparison between patients with normal and abnormal SPECT MPI and a comparison between patients with small and moderate/large perfusion defects.

shown in Table 1. Patients with abnormal SPECT MPI were older, more often were men and diabetic, had higher BMI, and more often had hypertension and an LBBB. Of the 920 patients with abnormal perfusion results, $558(61 \%)$ had small total perfusion defects and $362(39 \%)$ had moderate/large total perfusion defects. Of the 575 patients with ischemic defects, $353(61 \%)$ had small and 222 (39\%) had moderate/large ischemic perfusion defects. The baseline characteristics in patients with small and moderate/ large ischemic and total perfusion defects were similar, with the exception of a lower frequency in family history of CAD in patients with a moderate/large total perfusion defect and a higher frequency of LBBB in patients with a small ischemic defect (Table 1). The mean radiation dose was $6.5 \pm 3.5 \mathrm{mSv}$, and the mean administered dose of ${ }^{99 \mathrm{~m}} \mathrm{Tc}$-tetrofosmin was $736 \pm 410 \mathrm{MBq}$ for the entire cohort.

\section{Follow-up}

A total of 8 patients were lost to follow-up; the remainder of 4,049 patients $(99.8 \%)$ was included for the follow-up analysis. During a median follow-up of 2.4 y (25th-75th percentile, 1.73.4 ), a total of 37 primary events (14 cardiac deaths and 23 nonfatal MIs) and 116 secondary events (79 late revascularizations plus primary events) occurred. Unadjusted annual event rates for both outcome measures stratified by SPECT MPI findings are demonstrated in Figure 1. After multivariate analysis, LBBB, diabetes mellitus, and SPECT findings were significant independent predictors for cardiac death/MI (Fig. 2). Significant independent predictors for late revascularization/cardiac death/MI were age, male sex, diabetes mellitus, and SPECT MPI (Fig. 3). Adjusted survival curves based on ischemic and total perfusion defect for the primary and secondary outcomes are demonstrated in Figure 4. The risk of cardiac death/MI showed a significantly higher risk for both ischemic and total moderate/large perfusion defects. With respect to late revascularization/cardiac death/MI, a significant stepwise increase was present for patients with small ischemic or total perfusion defects and moderate/large ischemic or total perfusion defects.

\section{DISCUSSION}

Our study demonstrated that MPI acquired with the CZT SPECT camera has excellent prognostic value in patients suspected of having CAD. A normal MPI is associated with low event rates, and in abnormal MPI the extent of abnormality is associated with an increased risk of events.

SPECT MPI is well established for the diagnostic and prognostic evaluation of patients with suspected CAD. Novel cardiac $\gamma$-cameras using multipinhole collimation and solid-state detectors offer several advantages compared with the conventional SPECT cameras. Imaging time and radiation dose can be reduced, whereas diagnostic accuracy appears to be unchanged $(9,10,22)$. However, large clinical studies evaluating the prognostic value of SPECT imaging with CZT are scarce $(11,12)$.

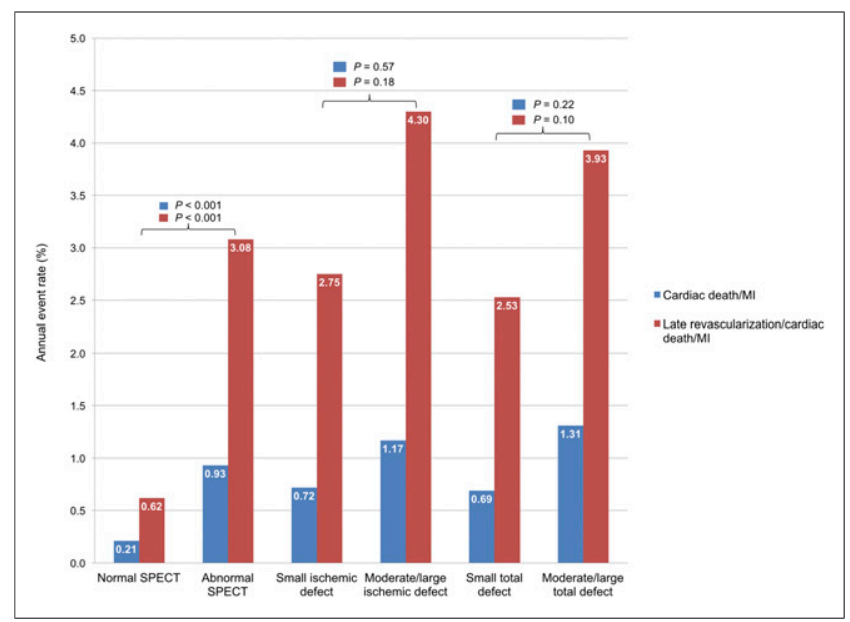

FIGURE 1. Unadjusted annual event rates stratified by SPECT MPI findings. 


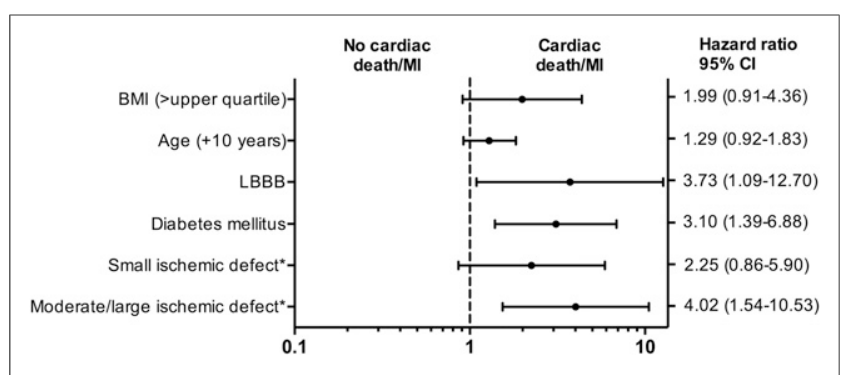

FIGURE 2. Multivariate cox survival analysis for cardiac death/MI. Variables sex, hypercholesterolemia, family history of CAD, current smoking, and hypertension were not included in multivariate analysis because these did not affect outcome measure or a $P$ value $>0.05$ in univariate analysis. ${ }^{*}=$ reference category, normal SPECT; $\mathrm{Cl}=$ confidence interval; upper quartile, 30 .

The present study, in the largest cohort of patients to date, shows a low event rate of both cardiac death/MI and late revascularization/cardiac death/MI in patients with normal myocardial perfusion $(0.21 \%$ and $0.62 \%$ annual risk, respectively). Patients with abnormal SPECT MPI findings had a significantly higher risk of events during follow-up, and with the extent of abnormality this risk increased. These findings demonstrate that MPI obtained with CZT SPECT is appropriate for the prognostic evaluation of patients suspected of having CAD. In a previous study performed in 1,613 patients on a CZT camera, annual event rates for all-cause death were $1.3 \%$ for normal SPECT and $5.6 \%$ for moderate/severely abnormal SPECT (12). Another study performed on a CZT camera in 1,109 patients described event rates for cardiac death/MI of $0.4 \%$ for patients with no significant perfusion abnormalities and $6.8 \%$ for patients with abnormal perfusion during a median follow-up of $1.7 \mathrm{y}$ (11). In both studies, a perfusion defect of greater than $10 \%$ of the myocardium was shown to be an independent predictor of hard events $(11,12,23)$. These results are similar to our findings, although lower annual event rates in our study were demonstrated in patients with abnormal SPECT. This annual event rate could be explained by the relatively low-risk population included in our study, because patients with a history of CAD were excluded. However, also in this low-risk population SPECT findings were independently associated with events. These findings are comparable with conventional SPECT cameras (23). More recently, Oldan et al. compared the

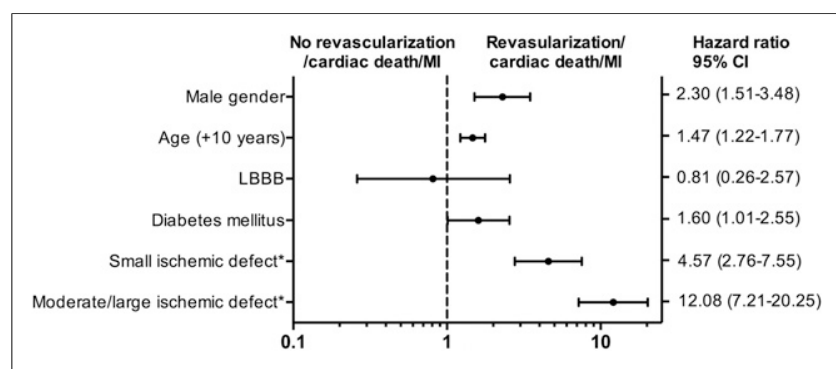

FIGURE 3. Multivariate cox survival analysis for late revascularization/ cardiac death/MI. Variables BMI, hypercholesterolemia, family history of CAD, current smoking, and hypertension were not included in multivariate analysis because these did not affect outcome measure or a $P$ value $>0.05$ in univariate analysis. ${ }^{*}=$ reference category, normal SPECT $; \mathrm{Cl}=$ confidence interval.

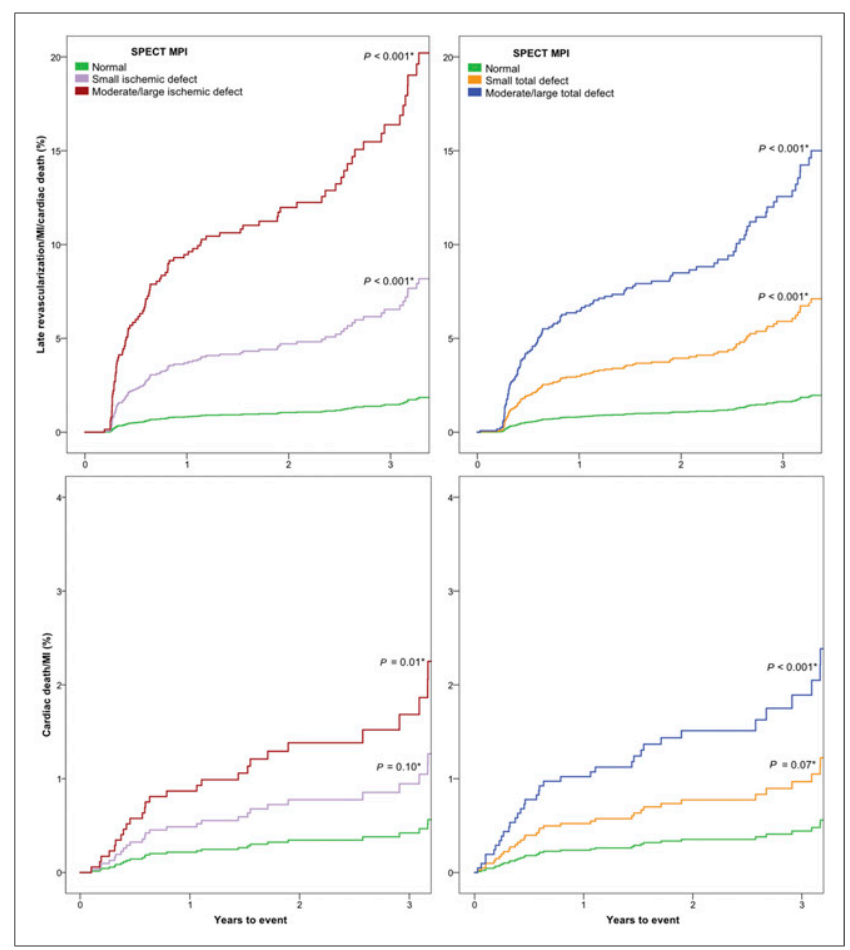

FIGURE 4. Adjusted survival curves of primary and secondary outcome measure for ischemic and total perfusion defects Cardiac death/MI curves adjusted for age, BMI, LBBB, and diabetes mellitus. Late revascularization/cardiac death/MI curves adjusted for age, sex, LBBB, and diabetes mellitus. ${ }^{*}=$ reference category, normal SPECT.

prognostic value of conventional and CZT camera in 2,088 patients and found that the extent of ischemia was associated with death and MI, without difference between camera type (24). It was also demonstrated that the prognostic value of normal SPECT imaging acquired with a CZT camera is excellent in obese patients (25).

With the excellent prognostic value of the CZT camera as demonstrated by our results and the previously described findings, future perspectives lie in lowering radiation dose and imaging time. These can be achieved by implementing a stress-first imaging protocol, in which rest imaging is omitted when the stress images are normal. These stress-only protocols are recommended for appropriately selected patients to lower radiation dose and imaging time (26). With CZT cameras, these stress-first imaging protocols could be more effectively implemented, because imaging results of the CZT camera are more often interpreted as normal (22). The prognostic value of the CZT camera has also been studied in stress-first imaging protocols. Einstein et al. studied 100 patients undergoing a low-dose CZT scan in the emergency department and demonstrated that the prognosis with a normal MPI was excellent. Yokota et al. demonstrated that the prognostic value of normal stress-only SPECT imaging was excellent in patients with CZT and conventional camera (cardiac events, $1.5 \% / y$ and $2 \% / y$, respectively; $P=0.08$ ) (27). In addition, the injected radiation dose could be lowered in patients undergoing CZT SPECT, because the heart-centric method of collimation of the CZT camera enhances count sensitivity (28). The effective radiation dose could be less than $1 \mathrm{mSv}$, without significant sacrifice of accuracy $(29,30)$. 
Although our study represents true daily practice and included consecutive patients, we acknowledge several limitations. The observational design remains a major limitation, because endpoints were not prespecified. Also, this is a single-center study in patients with suspected CAD and a low to intermediate pretest likelihood undergoing mainly pharmacologic stress. Therefore, results cannot be generalized to patient populations with different pretest likelihood or patients undergoing traditional exercise testing. Although we corrected for well-known factors that could induce nonischemic SPECT defects (obesity, LBBB), it cannot be excluded that other variables could cause nonischemic defects. Also, we did not perform follow-up for procedures that are generally not the result of MPI, such as non-bypass grafting cardiac surgery or implantable cardioverter defibrillator implantation. Moreover, we did not include the gated left ventricular function in the survival analysis. No comparison with conventional SPECT MPI was performed. Furthermore, CZT cameras differ significantly in design, and it cannot be excluded that different CZT cameras have different prognostic ability. Recent studies show that lowering the dose is feasible without negative impact on image quality $(29,30)$. However, this lower dose was not yet implemented in this study period.

\section{CONCLUSION}

Our findings show that MPI acquired with a CZT SPECT camera provides excellent prognostic information, with low event rates in patients with normal myocardial perfusion. In patients with abnormal SPECT MPI, the extent of abnormality is independently associated with an increased risk of events.

\section{DISCLOSURE}

No potential conflict of interest relevant to this article was reported.

\section{REFERENCES}

1. Underwood SR, Anagnostopoulos C, Cerqueira M, et al. Myocardial perfusion scintigraphy: the evidence. Eur J Nucl Med Mol Imaging. 2004;31:261-291.

2. Task Force Members, Montalescot G, Sechtem U, et al. ESC guidelines on the management of stable coronary artery disease: the task force on the Management of Stable Coronary Artery Disease of the European Society of Cardiology. Eur Heart J. 2013;34:2949-3003.

3. Fihn SD, Gardin JM, Abrams J, et al. ACCF/AHA/ACP/AATS/PCNA/SCAI/STS guideline for the diagnosis and management of patients with stable ischemic heart disease: a report of the American College of Cardiology Foundation/American Heart Association Task Force on practice guidelines, and the American College Of Physicians, American Association for Thoracic Surgery, Preventive Cardiovascular Nurses Association, Society for Cardiovascular Angiography and Interventions, and Society of Thoracic Surgeons. J Am Coll Cardiol. 2012;60:e44e164.

4. Hendel RC, Berman DS, Di Carli MF, et al. ACCF/ASNC/ACR/AHA/ASE/ SCCT/SCMR/SNM 2009 appropriate use criteria for cardiac radionuclide imaging: a report of the American College of Cardiology Foundation Appropriate Use Criteria Task Force, the American Society of Nuclear Cardiology, the American College of Radiology, the American Heart Association, the American Society of Echocardiography, the Society of Cardiovascular Computed Tomography, the Society for Cardiovascular Magnetic Resonance, and the Society of Nuclear Medicine. Circulation. 2009;119:e561-e587.

5. Iskandrian AS, Chae SC, Heo J, Stanberry CD, Wasserleben V, Cave V. Independent and incremental prognostic value of exercise single-photon emission computed tomographic (SPECT) thallium imaging in coronary artery disease. J Am Coll Cardiol. 1993;22:665-670.

6. Hachamovitch R, Berman DS, Shaw LJ, et al. Incremental prognostic value of myocardial perfusion single photon emission computed tomography for the prediction of cardiac death: differential stratification for risk of cardiac death and myocardial infarction. Circulation. 1998;97:535-543.

7. Oddstig J, Hedeer F, Jogi J, Carlsson M, Hindorf C, Engblom H. Reduced administered activity, reduced acquisition time, and preserved image quality for the new CZT camera. J Nucl Cardiol. 2013;20:38-44.

8. Duvall WL, Croft LB, Godiwala T, Ginsberg E, George T, Henzlova MJ. Reduced isotope dose with rapid SPECT MPI imaging: initial experience with a CZT SPECT camera. J Nucl Cardiol. 2010;17:1009-1014.

9. Duvall WL, Slomka PJ, Gerlach JR, et al. High-efficiency SPECT MPI: comparison of automated quantification, visual interpretation, and coronary angiography. J Nucl Cardiol. 2013;20:763-773.

10. Fiechter M, Ghadri JR, Kuest SM, et al. Nuclear myocardial perfusion imaging with a novel cadmium-zinc-telluride detector SPECT/CT device: first validation versus invasive coronary angiography. Eur J Nucl Med Mol Imaging. 2011;38:2025-2030.

11. Chowdhury FU, Vaidyanathan S, Bould M, et al. Rapid-acquisition myocardial perfusion scintigraphy (MPS) on a novel gamma camera using multipinhole collimation and miniaturized cadmium-zinc-telluride (CZT) detectors: prognostic value and diagnostic accuracy in a 'real-world' nuclear cardiology service. Eur Heart J Cardiovasc Imaging. 2014;15:275-283.

12. Nakazato R, Berman DS, Gransar H, et al. Prognostic value of quantitative highspeed myocardial perfusion imaging. J Nucl Cardiol. 2012;19:1113-1123.

13. Diamond GA, Forrester JS. Analysis of probability as an aid in the clinical diagnosis of coronary-artery disease. N Engl J Med. 1979;300:1350-1358.

14. Surawicz B, Childers R, Deal BJ, et al. AHA/ACCF/HRS recommendations for the standardization and interpretation of the electrocardiogram: part III-intraventricular conduction disturbances: a scientific statement from the American Heart Association Electrocardiography and Arrhythmias Committee, Council on Clinical Cardiology; the American College of Cardiology Foundation; and the Heart Rhythm Society. Endorsed by the International Society for Computerized Electrocardiology. J Am Coll Cardiol. 2009;53:976-981.

15. Henzlova MJ, Cerqueira MD, Mahmarian JJ, Yao SS. Quality Assurance Committee of the American Society of Nuclear Cardiology: stress protocols and tracers. J Nucl Cardiol. 2006;13:e80-e90.

16. Herzog BA, Buechel RR, Katz R, et al. Nuclear myocardial perfusion imaging with a cadmium-zinc-telluride detector technique: optimized protocol for scan time reduction. J Nucl Med. 2010;51:46-51.

17. Mouden M, Ottervanger JP, Timmer JR, et al. Myocardial perfusion imaging in stable symptomatic patients with extensive coronary atherosclerosis. Eur J Nucl Med Mol Imaging. 2014;41:136-143.

18. Berman DS, Abidov A, Kang X, et al. Prognostic validation of a 17-segment score derived from a 20-segment score for myocardial perfusion SPECT interpretation. J Nucl Cardiol. 2004;11:414-423.

19. Berman DS, Hachamovitch R, Kiat H, et al. Incremental value of prognostic testing in patients with known or suspected ischemic heart disease: a basis for optimal utilization of exercise technetium-99m sestamibi myocardial perfusion singlephoton emission computed tomography. J Am Coll Cardiol. 1995;26:639-647.

20. Radiation dose to patients from radiopharmaceuticals (addendum 2 to ICRP publication 53). Ann ICRP. 1998;28:1-126.

21. Thygesen K, Alpert JS, Jaffe AS, et al. Third universal definition of myocardial infarction. J Am Coll Cardiol. 2012;16:1581-1598.

22. Mouden M, Timmer JR, Ottervanger JP, et al. Impact of a new ultrafast CZT SPECT camera for myocardial perfusion imaging: fewer equivocal results and lower radiation dose. Eur J Nucl Med Mol Imaging. 2012;39:1048-1055.

23. Shaw LJ, Iskandrian AE. Prognostic value of gated myocardial perfusion SPECT. J Nucl Cardiol. 2004;11:171-185.

24. Oldan JD, Shaw LK, Hofmann P, et al. Prognostic value of the cadmium-zinctelluride camera: a comparison with a conventional (anger) camera. $\mathrm{J} \mathrm{Nucl}$ Cardiol. 2016;23:1280-1287.

25. De Lorenzo A, Peclat T, Amaral AC, Lima RS. Prognostic evaluation in obese patients using a dedicated multipinhole cadmium-zinc telluride SPECT camera. Int J Cardiovasc Imaging. 2016;32:355-361.

26. Cerqueira MD, Allman KC, Ficaro EP, et al. Recommendations for reducing radiation exposure in myocardial perfusion imaging. J Nucl Cardiol. 2010;17:709-718.

27. Yokota S, Mouden M, Ottervanger JP, et al. Prognostic value of normal stressonly myocardial perfusion imaging: a comparison between conventional and CZT-based SPECT. Eur J Nucl Med Mol Imaging. 2016;43:296-301.

28. Nichols KJ, Van Tosh A, Palestro CJ. Prospects for advancing nuclear cardiology by means of new detector designs. J Nucl Cardiol. 2009;16:691-696.

29. Nakazato R, Berman DS, Hayes SW, et al. Myocardial perfusion imaging with a solid-state camera: simulation of a very low dose imaging protocol. J Nucl Med. 2013;54:373-379.

30. van Dijk JD, Jager PL, Ottervanger JP, et al. Minimizing patient-specific tracer dose in myocardial perfusion imaging using CZT SPECT. J Nucl Med Technol. 2015;43:36-40. 